\title{
Determining the Effect of Deprivation on Quality of Life after Total and Unicompartmental Knee Arthroplasty
}

\author{
Abid Malik, Mudussar Abrar Ahmad, Justin Peter Cobb \\ Department of Orthopaedics, Charing Cross Hospital, London, UK \\ Email: abidmalikliverpool@hotmail.com
}

Received 10 May 2015; accepted 8 June 2015; published 11 June 2015

Copyright (C) 2015 by authors and Scientific Research Publishing Inc.

This work is licensed under the Creative Commons Attribution International License (CC BY). http://creativecommons.org/licenses/by/4.0/

c) (i) Open Access

\begin{abstract}
Determining the effect of deprivation on quality of life after total and unicompartmental knee arthroplasty Quality of life (QoL) following joint replacement is now a national priority, with every patient being assessed using the Oxford Knee Score. The current literature is conflicted as to whether deprivation has any effect on outcome after knee surgery. The type of surgery is another variable that may determine outcome. We set out to investigate which of these factors was a more powerful predictor of outcome. A prospective trial began where, 68 patients were telephoned, ( 30 TKA's and 38 UKA's). Each were asked a series of questions comprising the Oxford Knee Score (post operation), Imperial Knee Score (post operation), EuroQol-5D (pre and post operation) and individual deprivation questions. This was compared with the deprivation status using Townsend scores. A comparison of total and unicompartmental outcomes were also analysed including costeffectiveness. No correlation was found between outcome and Townsend scores $(p<0.05)$. Age was seen to be a significant indicator of pre surgical QoL. However, a large and significant difference was found between UKA and TKA when using the EQ-5D $(p<0.05)$ and the Imperial Knee Score $(p<0.09)$. This was not reflected in the Oxford Knee Score. UKA's were also found to be more cost-effective than TKA's. Deprivation has no major effect on the outcome of knee surgery in London. A clear difference in efficacy exists between TKA's and UKA's. Sensitivity of scores needs to be addressed.
\end{abstract}

\section{Keywords}

Deprivation, Arthroplasty, Quality of Life, Unicompartmental 


\section{Introduction}

Access to health care is a fundamental right and one measure of a functioning and developed society. Inequities in access exist on both a global and a national level. Deprivation in terms of social and economic factors has been shown to reduce access to health care and quality of life after surgery [1]. The NHS plan, sets out to address inequity in health care and service provision. To do this effectively, health planners need access to data on health related quality of life and deprivation for small areas of the UK [1]. Our study is based in Charing Cross Hospital in Hammersmith, London which is the 56th most deprived area in the United Kingdom, according to the Index of Multiple Deprivation 2008 [2]. This makes it an ideal area for the study of deprivation.

There has been a recent interest in the disparity between expectation, outcome and social deprivation after surgery. However, there is only one research paper that specifically compares knee replacement and social deprivation [3]. This study showed no statistically significant results which is at odds with recent studies who have shown an inequity in access to health care by those in lower socio-economic groups [4] [5]. These factors have been shown to effect health care and general wellbeing. The outcome measures in this study also lack validity and so further research in this area is justified.

Unicompartmental knee replacements have been shown by some to have superior outcomes in comparison to total knee replacements in selected patients, in both function and cost, yet they still only account for $7 \%$ of the knee arthroplasty figures nationally [5] [6]. Despite being unpopular nationally, a UKA is much cheaper than a TKA. The average cost being £1500 cheaper for a UKA [7] [8].

Age and gender have not been established as major prognostic indicators or limiting factors in knee replacement surgery, however age is known to be associated with lower access to health care [9]. The analysis of these factors will provided additional information on outcomes after surgery.

\section{Quality of Life and Outcome Measures}

Quality of life (QoL) can be described as "goodness of life” with "health related quality of life” being one aspect of this wider term. These measures are gaining popularity due to their use as end points in the evaluation of interventional outcomes. Current definitions vary amongst the health care community and the taxonomy has been well described [10]. It is therefore necessary to have an informal working definition for the purpose of this study. This will be based on the work of Testa and Simonson [11] who describe QoL as "the physical, psychological, and socialdomains of health, seen as distinct areas that are influenced by a person's experiences, beliefs, expectations, and perceptions.”

To fully evaluate each variable of QoL is a complex task and the measure of quality of life used must be heavily evaluated to justify its use and must fulfil the criterion of sensitivity, reliability responsiveness and validity.

Health related quality of life is notoriously difficult to measure [11]. The current literature in orthopedics uses the Nottingham Health Profile (NHP) [12] or the EuroQol (EQ5D) [13]. The Nottingham Health Profile is based on lay definitions of health. This is not an index of disease but how people feel when in a state of ill health. It is not intended for use as a measure of "health related quality of life" in isolation. It should be combined with other functional measures. NHP is generally considered as a shallow measure with some questions being seen as redundant by many of the participants. The NHP has good face, content and criterion validity with correlation to change in disease state. Predictive validity in relation to length of stay in hospital has also been established. However, some sections overlap. Overall, it can be seen as a simple and inexpensive measure. However, limited functional scores, a lack of a mental index of distress and the fact that it needs supplementation limits is use. It is also considered too short for split half reliability and is a highly skewed measure with minor changes in health not detected [14].

It is for these reasons that the EuroQol (EQ-5D) [13] measure of quality of life is used. This scale is designed to give a single index for each health state and gives a standardised, non disease specific measure for economic analysis. It is a simple survey that is designed for self evaluation. Although it is considered a good scale for brevity and simplicity issues remain over its comprehensiveness which results in loss of information and sensitivity.

Studies looking at the correlation between EQ-5D and SF-36 found a higher dependency in EQ-5D with regards to activities of daily living and have shown similar response rates [14]. The EQ-5D has shown to have 
high sensitivity in the low regions, measure subjective burden, is the most internationally validated measure with a low administrative cost. However, there seems to be some lack of correspondence between health state and descriptors, which reflects the brevity of the measure. The measure is highly skewed and has shown poor sensitivity particularly when researching disease-based outcomes. The thermometer scale has been shown to be biasing and the time referent is considered too short [14]. Despite these disadvantages some studies have shown its reliability and validity when compared to other measures such a SF-36 and the Health Utilities Index [14]. Due to the fact that a telephone survey is being conducted a need for brevity and ease of delivery is highly important with enough comprehensiveness to adequately assess quality of life, making the EQ-5D an ideal if brief measure.

Knee function was assessed via the Oxford Knee Score [15]. This is considered to be short, simple and validated. Many other scores are used for assessing knee function that are derived from clinical and radiological data. However, this may be different from the concerns of the patient. Therefore, a 12 point Oxford Knee score has been developed. This has been extensively reviewed and is seen as reliable and valid but some issues remain over its specificity, which may prove unreliable if a patient has pain from other causes [14].

The patient's outcome was also assessed using a system developed by Weiss et al. (2002) [16] known as the Total Knee Score. This assesses the patient's function using three lifestyle activities. The frequency of participation is combined with the importance and amount of interference with the activity.

The Total Knee Score is multiplied by the UCLA activity scale. This rates the amount of activity possible by the individual on a scale of 1 - 10 with 10 being the most active and able to participate in frequent, impact sports. This novel formula, weights the Total Knee Score, giving a unique measure of quality of life, which we have termed the Imperial Knee Score. This may give a more accurate picture of the patient's individual ability in the areas that are most important to them.

Currently, a number of methods for assessing deprivation exist. The majority assess "material deprivation” such as the Townsend Score [17] [18]. This is a frequently used measure of material deprivation. Townsend Scores are an aggregated and equally weighted measure, combining four aspects of material deprivation. This is based on Census data that can be inaccurate. It also does not indicate the proportion of people in an area that are deprived and is more useful for urban areas. The level of deprivation in each area is assessed both individually and via Townsend scores for each postcode giving information on an individual and electoral ward basis. Combining data derived from the 2001 Census with individual questions proves to be a comprehensive examination of deprivation [2].

Other scores such as the Jarman Index [19] were developed as a measure of Primary Care Trust workload and enabled additional payments to General Practitioners depending on the level of deprivation. However, this does not assess material deprivation and currently the only available scores are based on the 1981 Census and thus reduce accuracy. Scores such as the Index of Multiple Deprivation could have been used as they employ similar measures as the Townsend Score and correlate well [20] [21].

It is the purpose of this study to explore whether social deprivation affects health related quality of life (QoL) and knee function after knee replacement. In the current climate, the social divide is attracting media and political attention. The effect of this on orthopaedic outcomes is therefore particularly relevant.

Our primary hypothesis is thus that more deprived patients fare worse following knee arthroplasty.

Our secondary hypotheses are these:

1) That older patients have poorer access to health care, and fare worse after knee arthroplasty;

2) That unicompartmental knee arthroplasty is more effective and more cost effective than total knee arthroplasty;

3) That the imperial knee score is more sensitive than the oxford knee score in detecting significant differences in health related quality of life.

\section{Materials and Methods}

A retrospective, single centre trial was conducted over a period of 6 months which included 68 patients. Patients were included in the trial if they received either a TKA or UKA in the last 12 months. Patient characteristics are given in Table 1. The patients were recruited from both the private and NHS sectors to ensure a wide spread of deprivation. Implant choice was governed by the surgeon and aetiology varied.

Patients were contacted by telephone and the following was assessed: 
Table 1. Sample population characteristics.

\begin{tabular}{cccc}
\hline & & Outcomes & Mean \\
\cline { 2 - 4 } $\mathbf{N}$ & Total & Uni & 68 \\
Mean Age & 30 & 38 & 70.9 \\
Gender & 72.4 & 69.4 & 15.5 \\
Male & & & 19.5 \\
Female & 12 & 19 & \\
Outcome measure & 18 & 21 & 21.22 \\
Mean Oxford Knee Score & & & 73.27 \\
Mean Imperial Knee Score & & & 0.215 \\
Mean EQ-5D (pre op) & 22.3 & 20.13 & 0.68 \\
Mean EQ-5D (post op) & 32.4 & 40.87 & 0.45 \\
Mean EQ-5D (change) & 0.22 & 0.21 & 6.19 \\
Mean Total Knee Score & 0.59 & 0.77 & 6.53 \\
\hline
\end{tabular}

- Individual factors-age, gender and type of operation.

- The Oxford knee questionnaire (post operation).

- The Total Knee Questionnaire (post operation).

- EuroQoL-5D (pre and post operation).

- Individual deprivation questions.

Townsend scores were calculated based on the Census data for each postcode.

\subsection{Outcome Measures}

\subsubsection{EuroQol (EQ-5D)}

This essentially consists of two sections-the descriptive system and the EQ-visual analogue scale (EQ-VAS). The descriptive system assesses five dimensions of health: mobility, self-care, usual activities, pain/discomfort and anxiety/depression. Each dimension having three levels. The EQ-VAS records self-rated health on a vertical visual analogue scale $(0-100)$. This is combined into a single summary index by applying a formula that attaches a weight to each of the health states [21]. The weights are not equal. Pain is weighted the most followed by mobility, anxiety/depression, self-care and finally usual activities such as shopping or housework. This produces a range from -0.594 (a state worse than death) to +1 (a state of normality). Both pre and post-surgery quality of life is assessed.

\subsubsection{Townsend Score (2001)}

Townsend Scores range from -3.17 to 13.27 with 13.27 being the most deprived area. Measures of material deprivation are aggregated including unemployment, housing, over-crowding and car ownership. Each variable is attached the same weight. These are standardised and combined to produce a single index of deprivation based on each electoral ward. The overall score for Hammersmith is +1.8 indicating an area that is above the national average of deprivation.

\subsubsection{Individual Deprivation Questions}

Each individual was asked: Employment/previous employment, house ownership, number of rooms/people in each house and car ownership. This gave additional information on deprivation status. An additional question of time taken to receive the operation and reasons for delays was also asked to see if a disparity in waiting times existed and whether this has any correlation with outcomes. However, the data proved to be inconsistent and therefore was not included in the correlation analysis. However, all the available data was studied on an individual basis to see if this subjectively correlated with Townsend Scores.

\subsubsection{The Oxford Knee Score}

The Oxford Knee Score is a twelve question, quantitative measure of knee function [15]. There are five possible 
responses which are scored from 1 to 5 . These are summated giving 60 for the worse possible knee and 12 being the best, a table showing the interpretation of the Oxford knee Score is provided overleaf (Table 2). It is designed to be easy to administer and reduces observer bias.

\subsubsection{Imperial Knee Score (IKS)}

This is a combination of the Total Knee Score [16] and the UCLA activity scale. The subject is asked to choose three activities which are most important to them. The effect of the knee on these activities is assessed. Along with the amount of time they spend doing each activity and how much time they wish to spend on each activity. The result is a score of expectation from 1 - 10. The result is multiplied by the UCLA scoring system for activity. This gives a number from 1 - 10 depending on the level of a person's functional ability. 10 being able to frequently partake in high impact sports and 1 only being able to partake in a sedentary lifestyle. The result is the Imperial Knee Score, which is shown to be a sensitive marker for quality of life.

$$
\text { IKS }=(1 / 10(\text { frequency of participation } * \text { bother score } * \text { importance score }))+5 * \text { UCLA }
$$

\subsubsection{Cost-Effectiveness}

A detailed analysis of the cost effectiveness of each procedure was undertaken. This involved calculating the expected life years remaining for each individual using a life table, provided by the Office of National Statistics (2005) [2]. This was multiplied by the gain in quality of life to produce Quality Adjusted Life Years (QALY's). QALY's are a commonly used measure of effectiveness.

A cost effectiveness ratio was calculated by dividing the expected cost for each procedure (data gathered from Charing Cross Hospital) by the average QALY gained.

$$
\text { Cost }- \text { effectiveness }=\text { Cost of the procedure/Gain in QALY }
$$

\subsection{Individual Measures}

\section{Statistics}

A power calculation was performed using "DSS research" software. An analysis of 102 knees comparing TKA and UKA conducted by Newman et al. (1998) [5] was analysed. A post surgical outcome of 81.6 in the TKA group was found compared to 91.1 in the UKA group at the five year mark. This is comparable with the data from other groups such as Laurencin et al. (2001) [6] where a $29 \%$ difference exists. Using a statistical computer program the sample population can be estimated with a power of $80 \%$ and a $\mathrm{p}<0.05$.

Descriptive statistics were conducted using SPSS. Histograms and box plots were produced allowing for visual analysis of quality of life and knee function scores. These were further analysed using Pearson's correlation coefficient. This tests for linear correlation which is shown to be valid in this situation. Uni-variate analysis was conducted using a non-paired student t-test after normality was shown using a Kolmogorov-Smirnov test. Multiple linear regression analysis was also conducted on the independent variables as age, gender, type of operation and Townsend Score compared with each outcome measure (dependant variable). This finds whether a linear relationship exists between the response variable and several predictor variables. Multiple Regression correlation coefficients were also produced to assess the proportion of variability explained by the regression in the sample $\left(\mathrm{R}^{2}\right)$.

\begin{tabular}{|c|c|}
\hline Score & Grading \\
\hline Score 12 to 20 & May indicate satisfactory joint function. May not require any formal treatment. \\
\hline Score 21 to 30 & $\begin{array}{l}\text { May indicate mild to moderate knee arthritis. Consider seeing your family physician for an assessment and possible } \\
\text { x-ray. You may benefit from non-surgical treatment, such as exercise, weight loss, and /or anti-inflammatory } \\
\text { medication }\end{array}$ \\
\hline Score 31 to 40 & $\begin{array}{l}\text { May indicate moderate to severe knee arthritis. See your family physician for an assessment and X-ray. Consider a } \\
\text { consult with an Orthopaedic Surgeon. }\end{array}$ \\
\hline Score 41 to 60 & $\begin{array}{l}\text { May indicate severe knee arthritis. It is highly likely that you may well require some form of surgical intervention, } \\
\text { contact your family physician for a consult with an Orthopaedic Surgeon. }\end{array}$ \\
\hline
\end{tabular}

\section{Table 2. Interpretation of the oxford knee score.}




\section{Results}

Initial analysis of the data using a Kolmogorov-Smirnov test found a normal distribution attributable to the Townsend score (sig. 0.001), age (sig. 0.04), pre-operation EQ-5D (sig. 0.0001) and an equally represented gender distribution. We therefore showed a good spread of deprivation, age and quality of life before surgery within the sample population. This data is reflected in histogram of Townsend Scores provided (Figure 1). We also found this to be the case in all the post operative outcome measures.

We could not demonstrate either a positive nor negative relationship in any outcome measure compared to Townsend Scores (Table 3). Similarly we could not show a correlation between pre-operation EQ-5D and Townsend Score. We found age to be a significant variable in pre-operation EQ-5D and consequentially improvement in EQ-5D after surgery. We could not find a link between age or gender with any other outcome measure.

Our study demonstrated a significant difference between pre and post op EQ-5D (sig. 0.0001) regardless of type of surgery. We have found a large and significant difference in EQ-5D post surgery between UKA and TKA. UKA showing a higher quality of life after surgery. Our study shows the pre-operation EQ-5D is not significantly different between UKA and TKA's, neither is age, gender or change in EQ-5D following surgery. This shows equal level of morbidity, gender and age distribution between the TKA and UKA groups before surgery. The difference is not reflected in the Oxford Knee Score or the Total Knee Score but is reflected in both the EQ-5D (sig. 0.05) and the Imperial Knee Score (sig. 0.09) (Table 3). This may be attributable to the Oxford Knee Score clustering the results compared to the equally distributed Imperial Knee Score as shown in the histogram (Figure $2 \&$ Figure 3). Box plots showing the difference in scores pre and post surgery clearly show a benefit with UKA compared to TKA (Figure 4).

The final analysis is the cost effectiveness of each procedure. We find a cost per QALY of $£ 1160.93$ for a TKA compared with $£ 711.97$ for a UKA.

This demonstrates a £448.96 saving per quality of life year gained with a UKA compared with a TKA (Figure 5). This was based on the EQ-5D gain of quality of life being 0.52 for a UKA compared with a gain of only 0.36 with a TKA and the average cost being $£ 1500$ cheaper for a UKA.

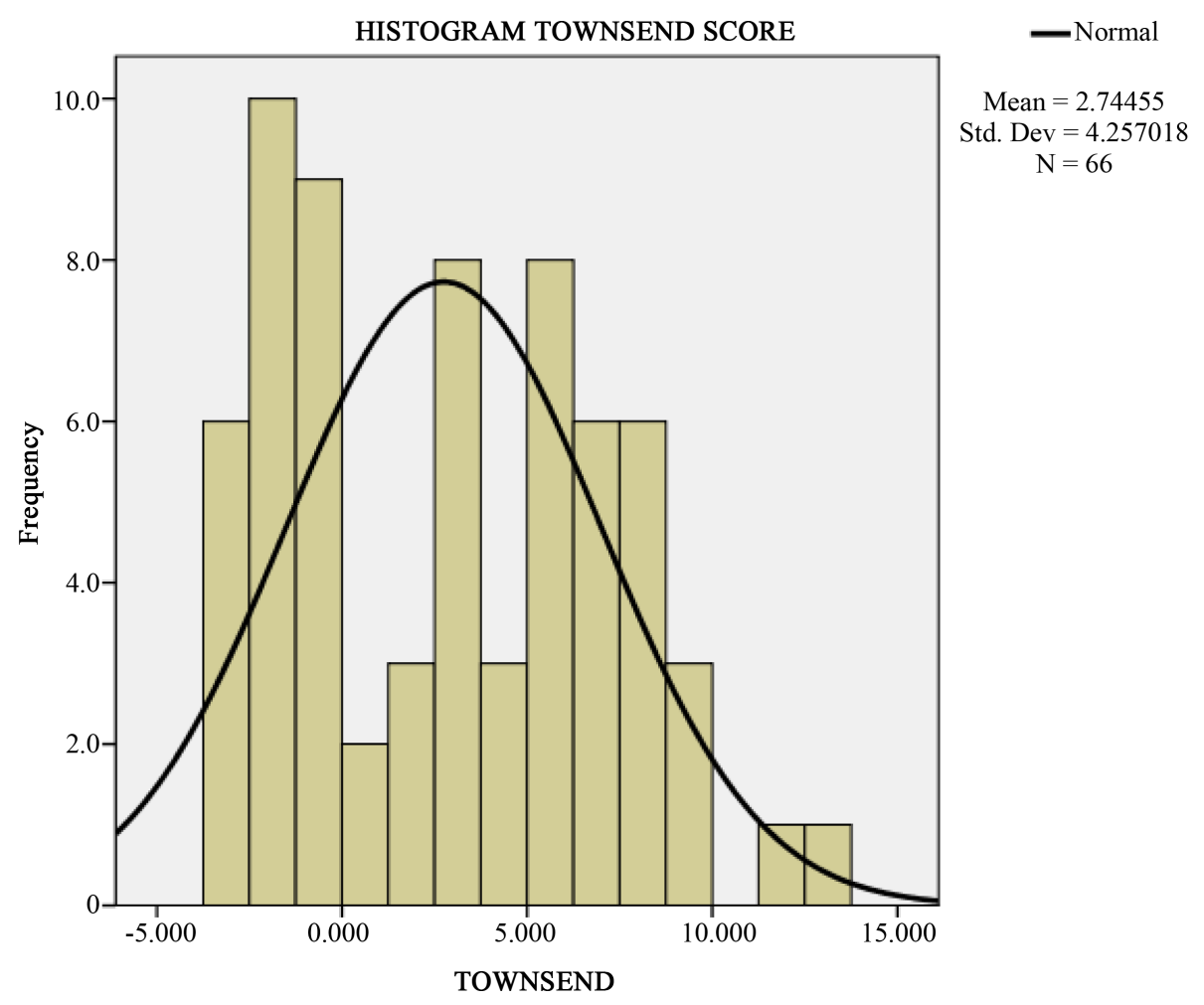

Figure 1. Normal distribution of Townsend scores. Footnote: Histogram showing normal distribution of deprivation in the sample population. 


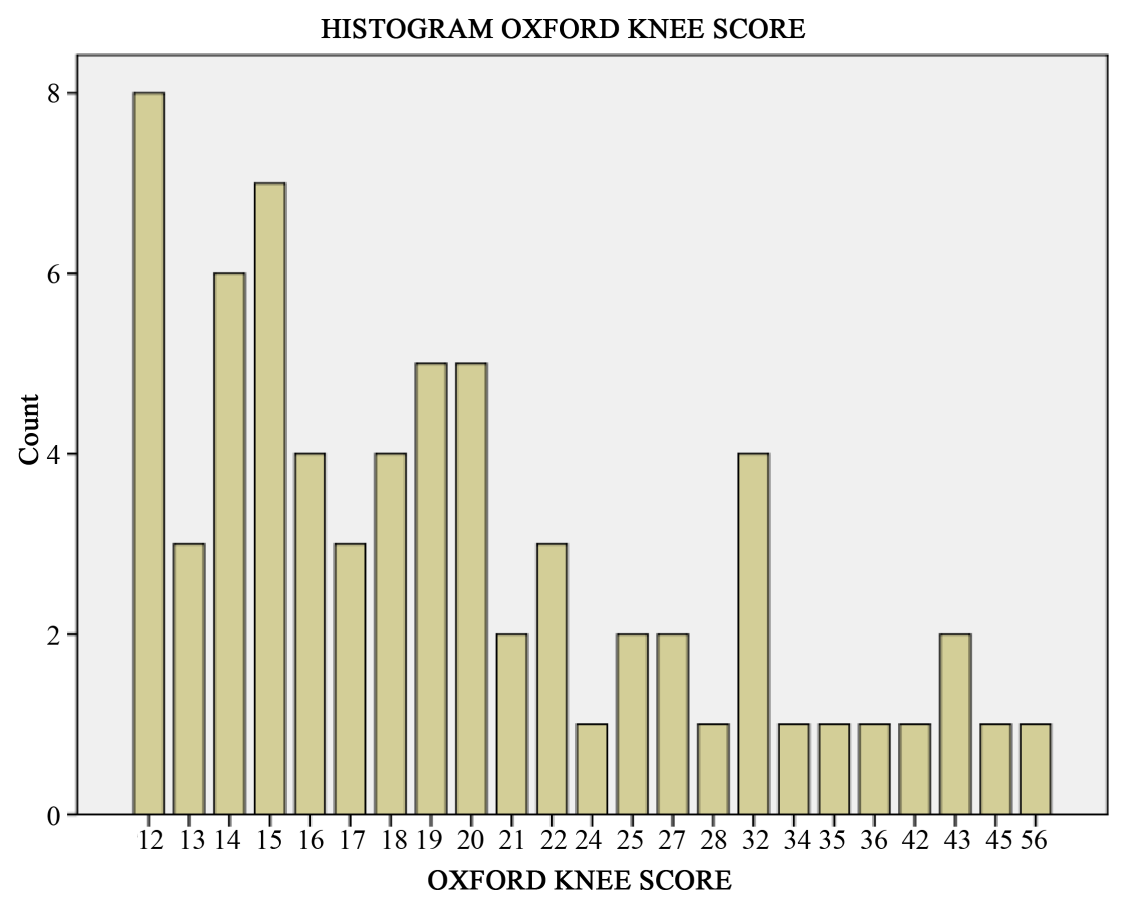

Figure 2. Oxford Knee score (compare with imperial Knee score Figure 3). Footnote: The histograms (Figure 2 \& Figure 3) show the clustering of data when using the Oxford Knee Score which is not reflected in either the Imperial Knee Score or the EQ-5D. The EQ-5D shows the highest sensitivity when comparing groups and this may be attributable to the weighting system.

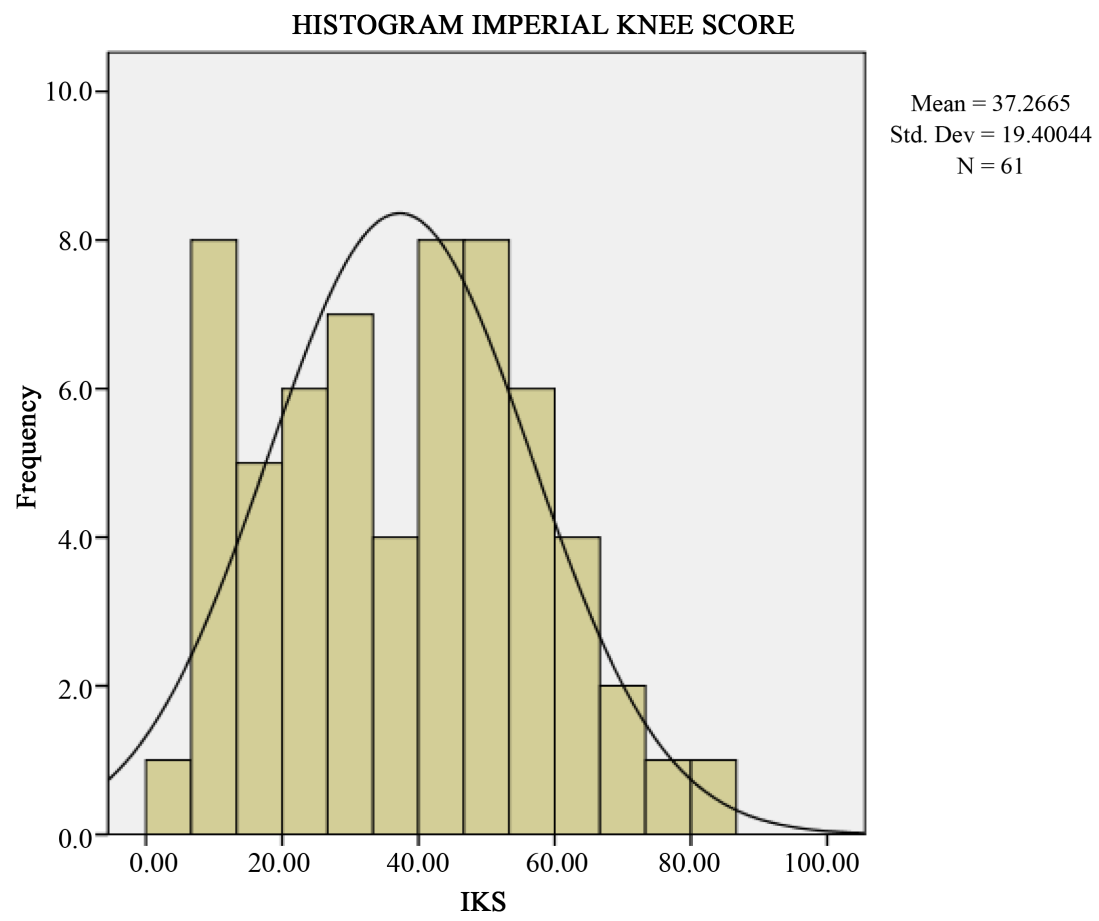

Figure 3. Histogram of imperial Knee score. Footnote: The histograms (Figure 2 \& Figure 3) show the clustering of data when using the Oxford Knee Score which is not reflected in either the Imperial Knee Score or the EQ-5D. The EQ-5D shows the highest sensitivity when comparing groups and this may be attributable to the weighting system. 


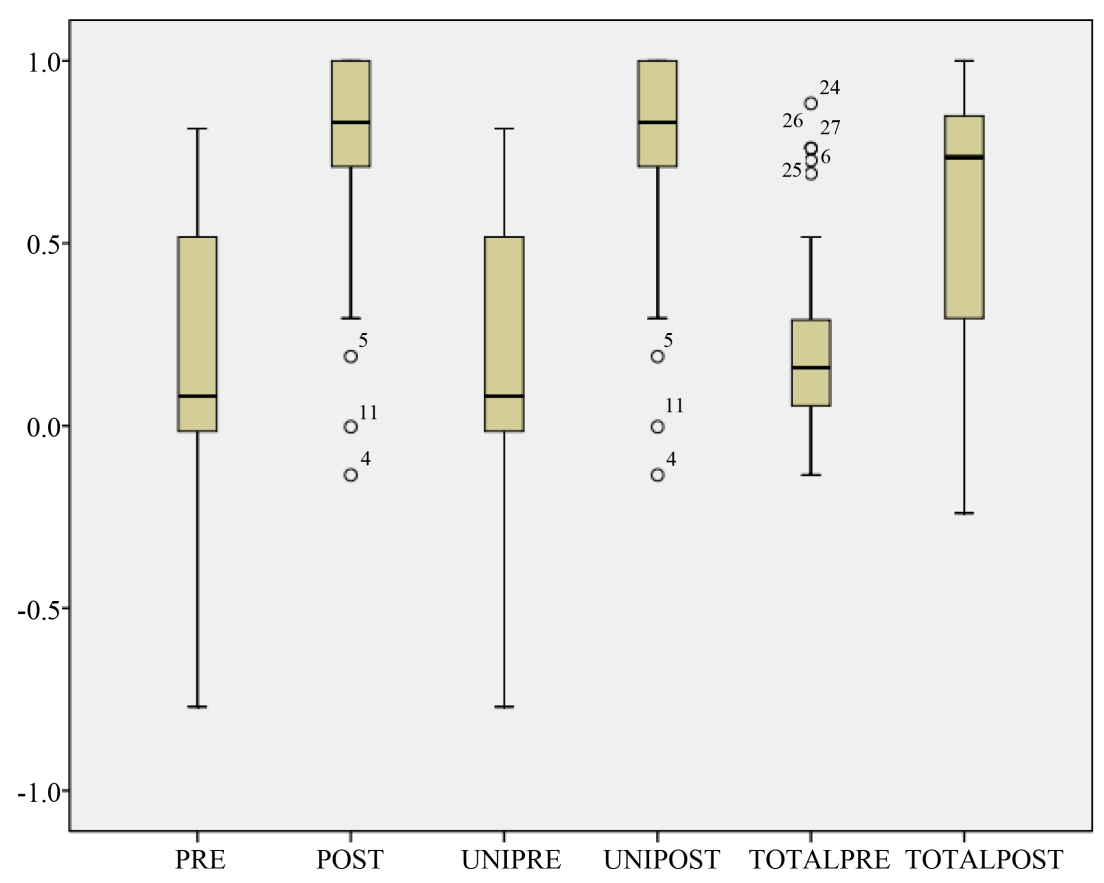

Figure 4. Box-plot of EQ-5D pre and post surgery and comparison of TKA and UKA.

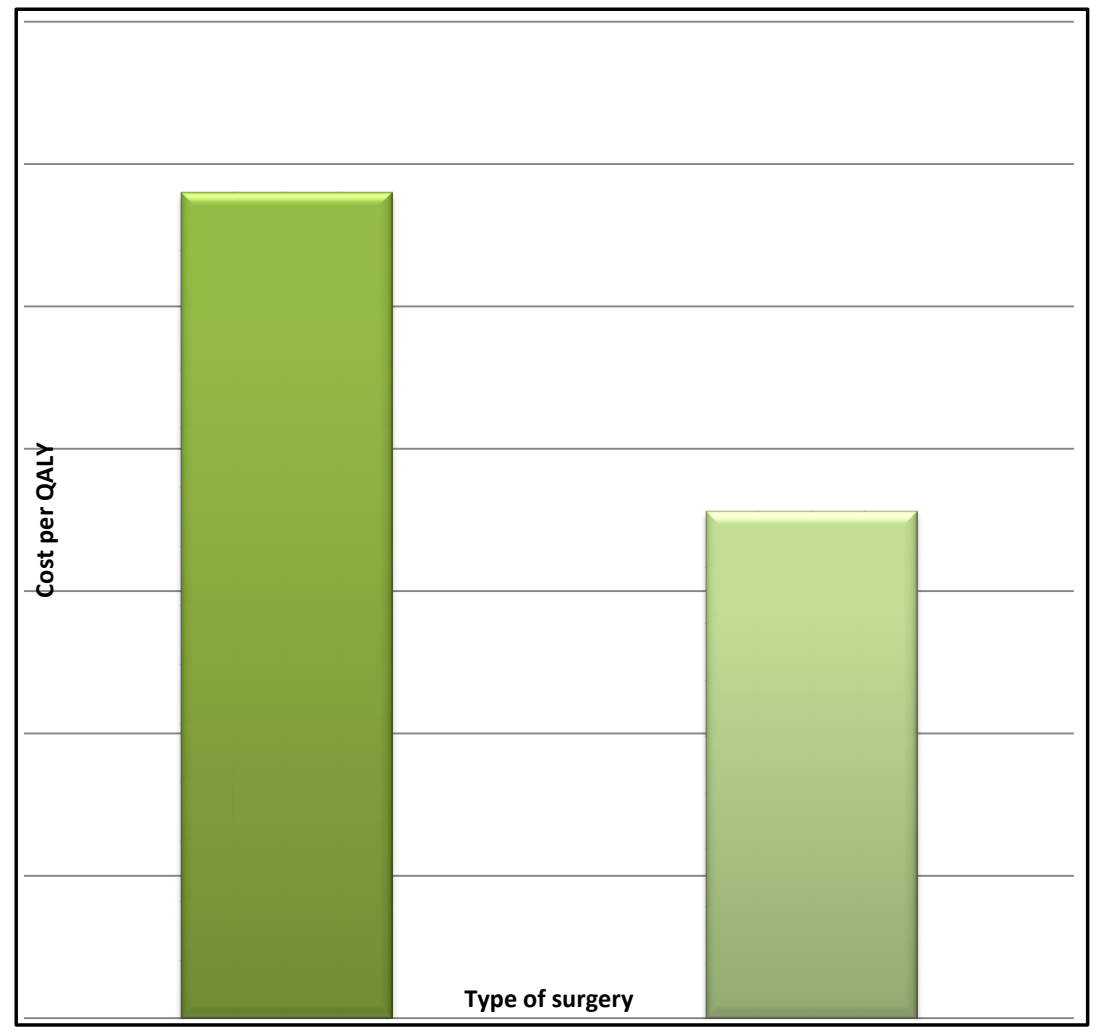

Figure 5. Cost-benefit analysis comparing UKA and TKA. Footnote: A fiscal benefit is demonstrated with a cost per quality of life year gained favouring the UKA. This is based on the change in QoL being 0.52 (uni-) compared with 0.36 (total). This is combined with the total cost of each operation which is set at $£ 5411$ for a UKA compared with £6956 for a TKA (Data from the UK National Joint Registry [8]) and the average life expectancy of both groups. This produces the quality of life years gained per cost. 
Table 3. Statistical results.

\begin{tabular}{|c|c|c|c|c|}
\hline Statistic & Pearson's & p-value & Regression & p-value \\
\hline \multicolumn{5}{|l|}{ Outcome vs. Townsend score } \\
\hline Oxford Knee Score & +0.122 & 0.33 & 0.953 & 0.345 \\
\hline Imperial Knee Score & -0.036 & 0.784 & -0.161 & 0.873 \\
\hline $\begin{array}{c}\text { Total Knee Score } \\
\text { EQ-5D }\end{array}$ & -0.084 & 0.526 & -0.66 & 0.512 \\
\hline \multicolumn{4}{|l|}{ EQ-5D } & \\
\hline (pre-op) & +0.044 & 0.723 & 0.074 & 0.941 \\
\hline (post-op) & -0.059 & 0.635 & -0.447 & 0.657 \\
\hline (Change) & -0.080 & 0.523 & -0.41 & 0.683 \\
\hline Unicompartmental cf. Total & Pearson's & p-value & Student t-test & p-value \\
\hline Oxford Knee Score & -0.111 & 0.369 & 0.902 & 0.370 \\
\hline Imperial Knee Score & 0.21 & $0.09^{*}$ & -1.724 & 0.09 \\
\hline Total Knee Score & 0.069 & 0.595 & 0.510 & 0.595 \\
\hline \multicolumn{5}{|l|}{ EQ-5D } \\
\hline pre-op & 0.02 & 0.874 & -0.163 & 0.874 \\
\hline post-op & 0.274 & $0.024^{* *}$ & -2.254 & $0.024^{* *}$ \\
\hline change & 0.193 & 0.115 & -1.616 & 0.115 \\
\hline Other significant results & & & Regression t value & p-value \\
\hline Age vs. Pre op EQ-5D & - & - & 2.4 & $0.019^{* *}$ \\
\hline Age vs. Change EQ-5D & - & - & -2.274 & $0.026^{* *}$ \\
\hline Uni vs. Total post op EQ-5D & - & - & 2.13 & $0.037^{* *}$ \\
\hline Pre vs post op EQ-5D & & & -8.44 & $0.0001^{* *}$ \\
\hline
\end{tabular}

*Suggests significance, ${ }^{* *}$ Significant at 0.05 .

\section{Discussion}

The study is limited by the small sample population. However, this study has still shown significant results and can be seen as a preliminary study that demands further investigation in the areas identified.

Errors may also have occurred as two separate data collectors telephoned patients. Therefore, an observer bias may have arisen. However, a reliability study was conducted using 80 observations with an overall $70 \%$ similarity in outcome. EQ-5D has an overall inter-rater bias of $0.69-0.94$, which is consistent with the reproducibility study conducted. Data entry may also be a source of error, this was verified by a random sample of 20 patients who were studied by an independent observer and a parallel of $97 \%$ was found. Limitations in terms of outcome measures are discussed in the introduction.

To address the primary objective of this study i.e. to see if deprivation has an effect on outcome after knee surgery, our study failed to demonstrate a relationship between deprivation and any outcome measure. This can be seen to compliment the research by Murray et al. (2005) [3] who came to the same conclusion. The initial pre operation severity was also not linked to deprivation status which is at odds with the study conducted by Yong et al. (2004) [4] that states that deprivation may affect access to health care and hence severity at presentation to surgery. In addition, this study shows that age can be seen as a significant indicator of pre operation quality of life; however, this is not an indicator of outcome after surgery. A campaign to target the elderly with knee problems to seek advice on replacements may be warranted.

Knee replacement regardless of type of operation can be seen to improve quality of life ( $<<0.0001)$. However, we found a large and significant difference exists between the UKA's and TKA's after surgery. This can be seen as comparable to the studies by Laurencin (1996) and Newman (1998) [5] [6]. However, this improvement is only seen in the EQ-5D measure of quality of life and is suggested in the Imperial Knee Score, it is not reflected in either the Total Knee Score or the Oxford Knee Score. Showing the sensitivity of the IKS and EQ-5D. Both the EQ-5D and the Imperial Knee Score use a weighting system attached to each dimension of health and this may bestow the high degree of sensitivity. This is reflected in the normal distributions attributed to both the Imperial Knee Score and EQ-5D but clustering of data in the Oxford Knee Score.

Therefore, we can see that the National Health Service provider in the Hammersmith area is providing a high level of satisfaction to all clients regardless of social and economic background. Analysis at Charing Cross has 
revealed UKA to be indicated for the majority of patients with TKA being reserved for the minority. Expenditure in knee surgery in the UK is set at 300 million pounds with 93\% being TKA (UK National Joint Registry). This study has identified a $£ 448.96$ pound saving per quality of life year gained in using a UKA in comparison to a TKA. If this data was utilised and UKA replacement was used in $50 \%$ of the cases an overall saving of $£ 37$ million pounds and a cost utility gain of $£ 11$ million is identified.

If this is compared to the cost-effectiveness of other interventions such as renal dialysis which is estimated at $£ 30,000$ per QALY gained or chemotherapeutic drugs such as anastrazole being £17,656 per QALY gained UKA can be considered to be a relatively inexpensive and highly effective intervention [22]. In the current political and economic climate this can be seen as especially relevant.

\section{Conclusions}

In conclusion, in this study deprivation can be seen to have no effect on either access to or outcome after knee replacement. This has been identified in all outcome measures and upholds the principles of the NHS to provide an equal service to all regardless of economic status.

A clear difference in efficacy between TKA's and UKA's has been found in this study. Currently, the UKA knee has fluctuated in popularity; however, with the development of minimally invasive procedures and research into its benefits over TKA, it is the recommendation of this study to promote UKA with equal reflection in the monetary policy of the NHS. This will be both beneficial for the consumer and the NHS.

Finally, the sensitivity of scores for subjective rating of health related quality of life, needs to be addressed. We have shown that the Oxford Knee Score although considered a reliable scoring system and one of the most popular tools in orthopaedics, does not adequately reflect the multidimensional nature of quality of life. This study has shown that the EQ-5D and the Imperial Knee Score are much more sensitive in discriminating post surgical outcomes. The Imperial Knee Score can be seen as a sensitive and specific tool for measuring knee function and warrants further promotion and validation.

\section{References}

[1] Department of Health (2000) The NHS Plan. HMSO, London.

[2] Office National Statistocs (2005) Life Tables. http://www.statistics.gov.uk/STATBASE/ssdataset

[3] Murray, J., Birdsall, D.P., Cleary, R., Deehan, D.J. and Sher, J.L. (2005) Deprivation and Outcome after Knee Surgery. Journal of Bone and Joint Surgery, The Knee, 13, 98-101. http://dx.doi.org/10.1016/j.knee.2005.11.002

[4] Yong, P.F.K., Milner, P.C., Payne, J.N., Lewis, P.A. and Jennison, C. (2004) Inequalities in Access to Knee Joint Replacements for People in Need. Annals of the Rheumatic Diseases, 63, 1483-1489. http://dx.doi.org/10.1136/ard.2003.013938

[5] Newman, J.H., Ackroyd, C.E. and Shah, N.A. (1998) Unicompartmentalor Total Knee Replacement? Five-Year Results of a Prospective, Randomised Trial of 102 Osteoarthritic Knees with Unicompartmental Arthritis. Journal of Bone \& Joint Surgery, 91-B, 52-57.

[6] Laurencin, C.T., Zelicof, S.B., Scott, R.D. and Ewald, F.C. (1996) Unicompartmental versus Total Knee Arthroplasty in the Same Patient. Clinical Orthopaedics \& Related Research, 273, 151-156.

[7] Rasanen, P., et al. (2007) Effectiveness of Hip or Knee Replacement Surgery in Terms of Quality-Adjusted Life Years and Costs. Acta Orthopaedica, 78, 108-115. http://dx.doi.org/10.1080/17453670610013501

[8] National Joint Registry (2006) Knee Replacement Statistics. www.njrcentre.org.uk

[9] Ethgen, O., et al. (2004) Health-Related Quality of Life in Total Hip and Total Kne Arthroplasty. A Qualitative and Systematic Review of the Literature. Journal of Bone and Joint Surgery, 86, 963-974.

[10] Farquhar, M. (1995) Definitions of Quality of Life: A Taxonomy. Journal of Advanced Nursing, 22, 502-508. http://dx.doi.org/10.1046/j.1365-2648.1995.22030502.x

[11] Testa, M.A. and Simonson, D.C. (1996) Assessment of Quality-of-Life Outcomes. New England Journal of Medicine, 334, 835-840. http://dx.doi.org/10.1056/NEJM199603283341306

[12] Hunt, S.M., McEwen, J. and McKenna, S. (1986) Measuring Health Status. Croom Helm, London.

[13] The EuroQol Group (1990) EuroQol—A New Facility for the Measurement of Health-Related Quality of Life. Health Policy, 16, 199-208. http://dx.doi.org/10.1016/0168-8510(90)90421-9

[14] Bowling, A. (2005) Measuring Health: A Review of Quality of Life Measurement Scales. Open University Press, 
Berkshire.

[15] Orthopedic Scores (2006) Oxford Knee Score. http://www.orthopaedicscore.com/

[16] Weiss, J.M., Noble, P.C., Conditt, M.A., Kohl, H.W., Roberts, S., Cook, K.F., Gordon, M.J. and Mathis, K.B. (2002) What Functional Activities Are Important to Patients with Knee Replacements. Clinical Orthopaedics and Related Research, 172-188.

[17] Townsend, P. (1987) Deprivation. Journal of Social Policy, 16, 125-146. http://dx.doi.org/10.1017/S0047279400020341

[18] Townsend, P., Phillimore, P. and Beattie, A. (1988) Health and Deprivation: Inequality and the North. Croom Helm, Bristol.

[19] Jarman, B. (1984) Underprivileged Areas: Validation and Distribution of Scores. British Medical Journal, 289, 15871592. http://dx.doi.org/10.1136/bmj.289.6458.1587

[20] Cotterill, S. (2005) A Comparison of Deprivation Indices: Townsend 4 and Index of Multiple Deprivation 2004. Key Health Data for the West Midlands, University of Birmingham, Birmingham.

[21] Dolan, P. (1996) Modelling Valuations for Health States: The Effect of Duration. Health Policy, 38, 189-203. http://dx.doi.org/10.1016/0168-8510(96)00853-6

[22] Mansel, R., Locker, G., Fallowfield, L., Benedict, A. and Jones, D. (2007) Cost-Effectiveness Analysis of Anastrozole vs. Tamoxifen in Adjuvant Therapy for Early Stage Breast Cancer in the United Kingdom: The 5-Year Completed Treatment Analysis of the ATAC ("Arimidex", Tamoxifen Alone or in Combination) Trial. British Journal of Cancer, 97, 152-161. http://dx.doi.org/10.1038/sj.bjc.6603804 